\title{
ULF/ELF Plasma Waves in the Active Regions of the Magnetosphere
}

\author{
J. BLĘCKI \\ Space Research Centre Polish Academy of Sciences, Bartycka 18A 00716 Warsaw, Poland
}

(Received January 12, 1995; Revised October 25, 1995; Accepted November 14, 1997)

\begin{abstract}
The bow shock, magnetopause, plasma sheet and plasma sheet boundary layer are the regions in the plasma environment of the Earth, where intensive conversion of energy takes space. In present paper these regions are called the active regions. Low frequency plasma waves which can control the dynamics of the ions in collision free plasma play very important role in the formation and behaviour of these regions. This paper presents review of the observations of low frequency plasma waves $(0.1-105 \mathrm{~Hz})$, which were done by the PROGNOZ-8 satellite in the vicinity of the bow shock, at the dayside and nightside magnetopause within the flux transfer events and in the magnetotail. Common features of the studied regions are accelerated plasma streams, hot electron populations and very strong wave activity, particularly at the low frequencies. The detailed study of the wave spectra together with the distribution function for electrons indicate the correlation between presence of the lower-hybrid waves and the population of the particles with higher energy then in the surrounding space. The orbit of PROGNOZ-8 (apogee $32 R_{E}$ inclination $65^{\circ}$ ) allows us to study regions where the ISEE-1, 2 and AMPTE satellites did not operate.
\end{abstract}

\section{Introduction}

The energy transfer from the solar wind into the earth environment is the most intense at the bow shock, magnetopause and in the magnetotail. These regions are also associated with the strong wave activity. The question on the role of these waves is still open. This is a problem of "chicken and egg". How can one distinguish whether the observed phenomena in the discussed regions are cause or effect of one another? The discussion on the role of the plasma waves in anomalous transport in the magnetospheric plasma has a long history (Treumann et al. (1995) and reference therein).

The goal of this paper is to give some contribution to this subject reviewing the results of plasma wave measurements which have been performed by PROGNOZ-8 satellite in the regions of the magnetosphere which are called here active regions.

PROGNOZ-8 satellite, was launched in December 1980 into a highly elliptical orbit with apogee about $30 R_{E}$ and equatorial inclination $65^{\circ}$. The rotation period of the satellite was about $120 \mathrm{sec}$. To study the electric component of plasma wave we have used the double probe as a sensor and an onboard spectrum analyser which measured the spectral densities in the range $2-105 \mathrm{~Hz}$, at 9 frequencies. Bandwidth of every spectral line is $2 \mathrm{~Hz}$. Full spectrum was obtained every $30 \mathrm{sec}$. Once per orbit the wave form with sampling frequency $150 \mathrm{~Hz}$ have been transmitted. For plasma diagnostic we used the data from Swedish experiment Promics-2. It consisted energetic-ion composition spectrometer covering energy from 0.02 to $31 \mathrm{keV}$ with 32 steps, electron spectrometer with energy range $0.03-47 \mathrm{keV}$, spectrometer for protons and $\alpha$-particles with energies $0.27-4.4 \mathrm{keV}$ in 16 levels. Promics- 2 had 2 modes of operation-Solar wind mode with time resolution about $328 \mathrm{sec}$., Magnetospheric mode with time resolution about $41 \mathrm{sec}$.

\section{Bow Shock}

The collisionless bow shock is the place where supersonic solar wind plasma flow is stopped and energy of the flow is transformed into the thermal energy. For the collisionless plasma Sagdeev (1966) discussed the role of cooperative microprocesses in formation of collisionless bow shock. In this 


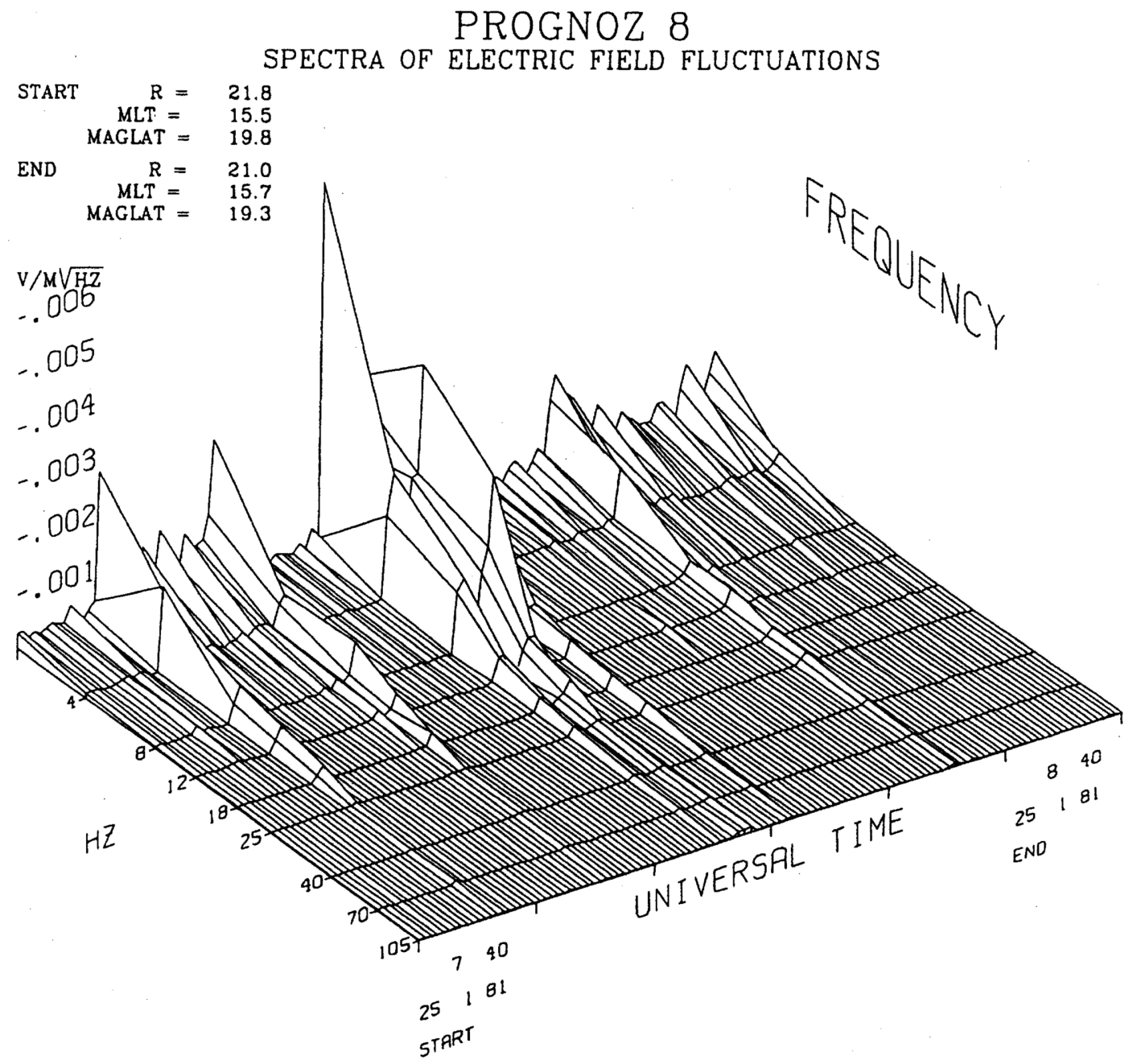

Fig. 1. Plasma wave spectra registered in the solar wind on 25 January, 1981. Vertical axis is spectral density of the electric field fluctuations, horizontal are frequency and time. The first burst of the wave activity (7:47 UT) is associated with the ion beam (see Fig. 2), the HFA was encountered at 8:07 UT. The characteristic maxima observed within the HFA at 4-12 $\mathrm{Hz}$ are associated with the lower-hybrid mode.

mechanism the microturbulence plays the role of collisions. PROGNOZ-8 detected at the bow shock lower hybrid waves and it has been found that these waves play very important role in electron heating (Vaisberg et al., 1983).

Here we focus on the another events close associated with the bow shock. The measurements done by ISEE-1 and 2, AMPTE-UKS and IRM satellites in the vicinity of the Earth bow shock in the solar wind and within the magnetosheath indicate existence of the small scale unusual plasma structures. These phenomena are characterised by very short duration (1-10 min), large plasma temperature increases, deflection of plasma flow and decreases of flow velocity, magnetic field enhancement at the boundary of the event (Thomsen et al., 1986; Woolliscroft et al., 1986; Paschmann et al., 1988; Schwartz et al., 1988). These structures have been called "hot flow anomalies". Mentioned satellites had the orbits with the apogee in the regions close to the bow shock in the equatorial plane. The examination of data from 
PROGNOZ-8 SEANS 039SW-MODE START DATE: $81-01-25$

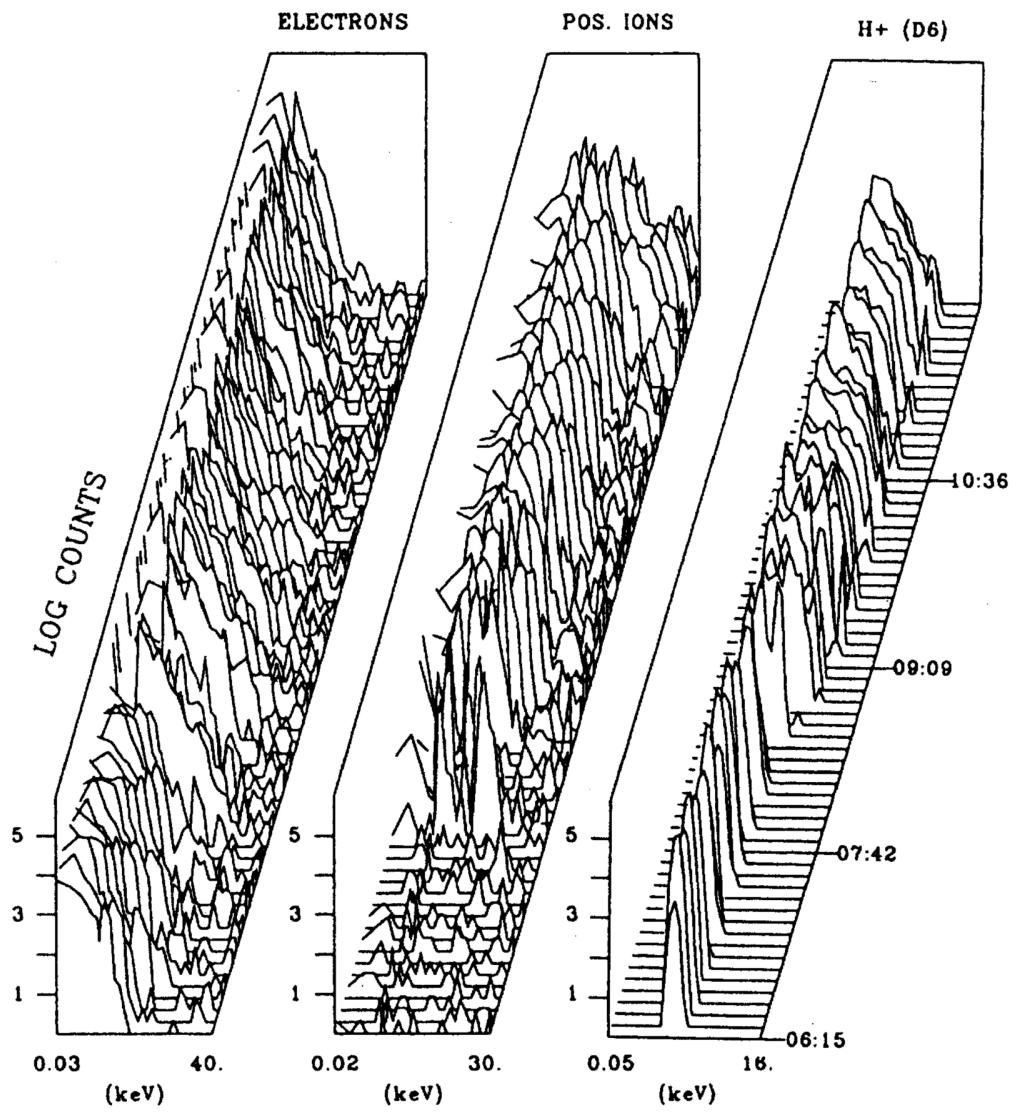

Fig. 2. Plasma particle energetic spectra obtained from Promics-2 experiment aboard the PROGNOZ-8 satellite for time interval 25 January 6:15-11:45 UT. The positive ion beam is seen at 7:47 UT. The broadening of the electron and ion spectra appears at 8:07 when encounter with the HFA took place.

PROGNOZ-8 shows, that similar structures can occur also at higher latitude and at greatest distances reached by PROGNOZ-8.

Figures 1 and 2 show the example of wave and plasma spectra taken within the event for January 25 , 1981, when the satellite was in the solar wind, at 7:47 UT the beam of the positive ions was registered (Fig. 2) the enhancement of the wave activity at this moment can be seen (Fig. 1). Few minutes later-7:56 UT the broadening of the electron distribution function was accompanied by strong wave activity at lower hybrid frequency $8 \mathrm{~Hz}$ (Figs. 1 and 2). Next region of hot electrons and strong wave activity was encountered at 8:07 UT. Characteristic maximum in wave spectra at 4-18 Hz again associated with lowerhybrid mode. The bow shock was crossed at 8:37 UT. Similar structures have observed by PROGNOZ8 at larger distances from the bow shock and within the magnetosheaths as well (Błęcki et al., 1993).

\section{Magnetopause}

The magnetopause is characterised very well by its wave activity (Anderson et al., 1982). During 9 months of the life time of PROGNOZ-8 more than 60 magnetopause crossings have been registered. Some of them in the real time transmission mode. The main result of these measurements is estimation that the 


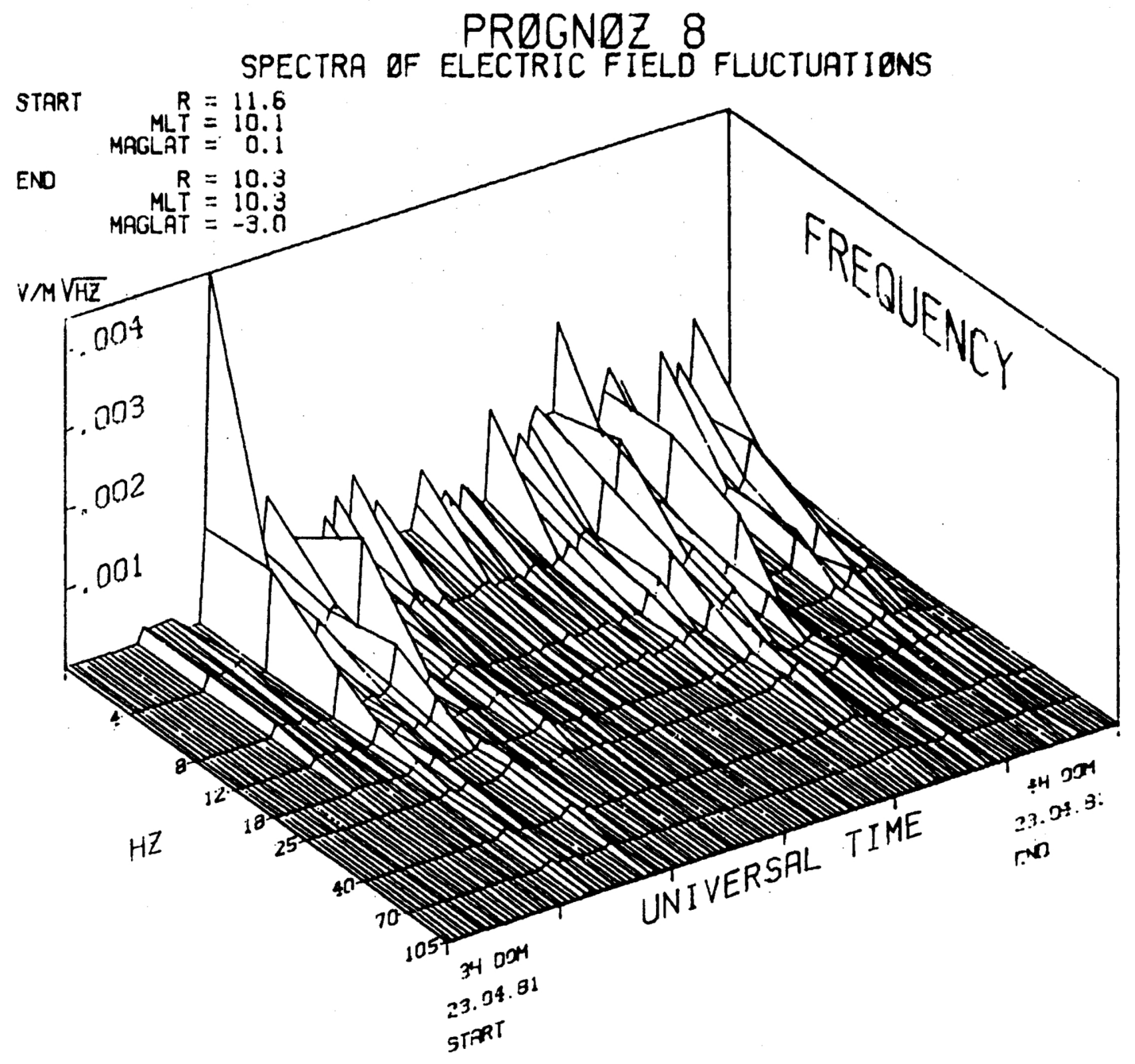

Fig. 3. The same as Fig. 1 for two flux transfer events (3:12 and 3:42 UT) at the dayside magnetopause.

permanent feature of the magnetopause is existence of ion-cyclotron and lower-hybrid waves at it (Blęcki et al., 1987, 1988; Belova et al., 1991).

As in the case of the bow shock the small scale structures have been also observed close to the magnetopause by the ISEE and AMPTE satellites. Main feature of these formations was special behaviour of the normal to the magnetopause component of the magnetic field (Russell and Elphic, 1979). These phenomena are known as the "flux transfer events".

The orbit of PROGNOZ-8 gave the chance to look for these events at high latitude magnetopause, which was not penetrated before. The set of observations can be divided into two parts-dayside and nightside observations. Figure 3 presents wave spectra for dayside event taken by onboard analyser. The maxima at $10-25 \mathrm{~Hz}$ are associated with $\mathrm{LH}$ frequency. As one can see the spectra change from one to another. It can be a signature of the nonstationarity of the processes. To study this problem we used the wave form obtained during the real time transmission. Figure 4 contains the spectra obtained by processing of wave form using two methods-fast Fourier transform (dashed line) and maximum entropy method 


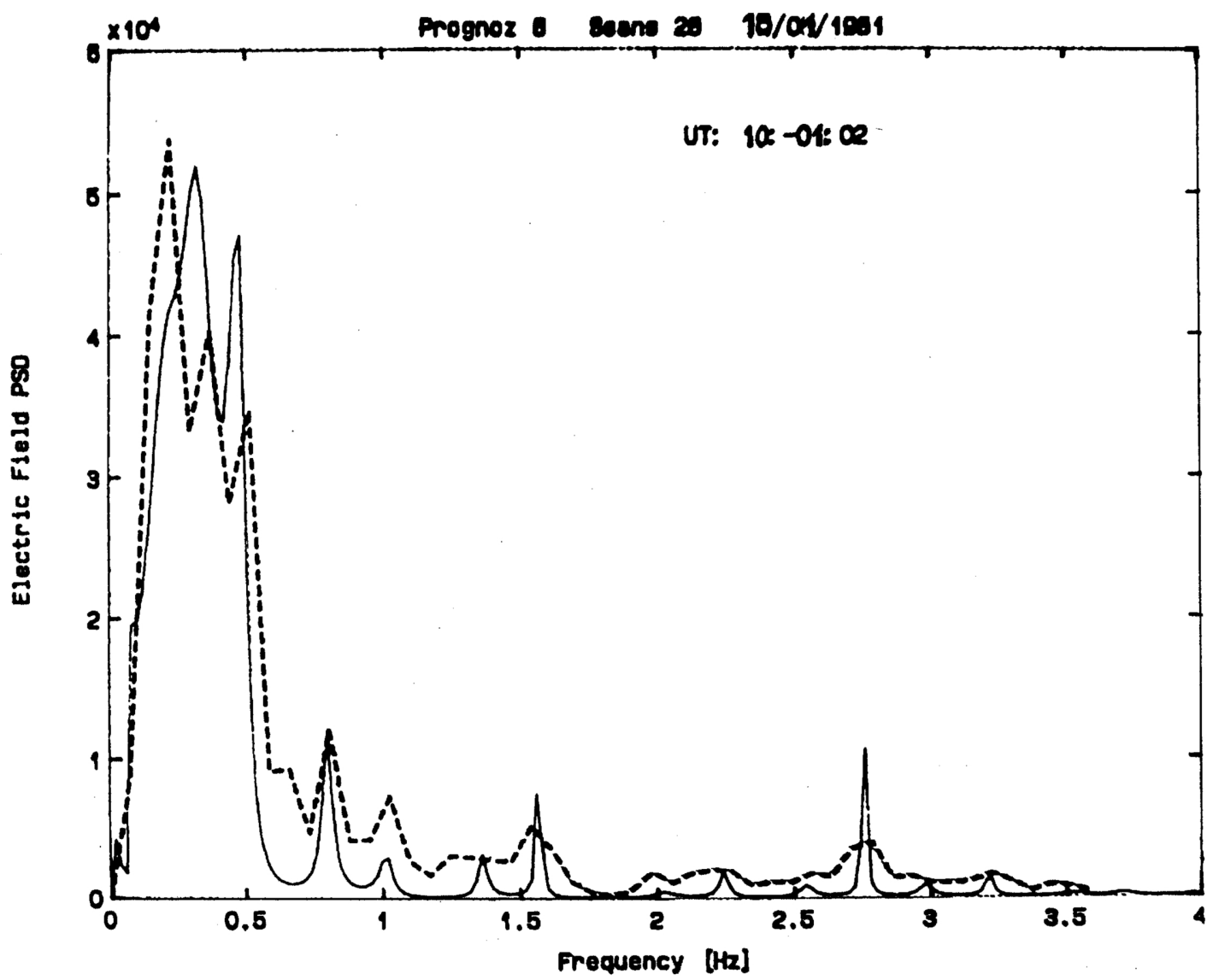

Fig. 4. Wave spectra obtained by the processing of the wave form. Two methods have been used-Fast Fourier transform (dashed line) and Maximum entropy method (solid line). Presented spectra were obtained for the leading edge of the FTE. The first maximum is associated with the ion-cyclotron frequency, next are harmonics of it.

(solid line) (Błęcki et al., 1990). The maxima given by both methods are very close each other and occur at the ion-cyclotron frequency and its harmonics.

During the last 4 months of the life time of PROGNOZ-8 more than 20 nightside magnetopause crossings were registered. Dense magnetosheath-like plasma clouds apparently detached from the magnetopause were often encountered by PROGNOZ-8 near the high latitude nightside magnetopause.

It was found that such structures are always associated with strong wave activity at the ion-cyclotron and lower-hybrid frequencies. Some observed clouds had bulk velocity higher than in nearby magnetosheath. More frequently, high energy non-maxwellian tails or high energy cold ion beams were observed. Plasma and magnetic field characteristics are similar to those in the flux transfer events observed on the dayside (Blęcki et al., 1987, 1988, 1990).

Figure 5 shows the example of plasma wave and plasma parameters for the magnetopause and MSlike plasma cloud crossing (27 July, 1981). PROGNOZ-8 passed through the magnetopause at 00:08 UT (dashed vertical line) and reached the region of intense plasma flux a few minutes later at 0:21 UT. An increasing of the wave activity occurs at the magnetopause and in discussed region as well. Figure 6 contains the detail electric field fluctuation spectra in the frequency range 2-105 Hz. Characteristic maxima at 25 and $40 \mathrm{~Hz}$ are associated with lower hybrid mode. The results obtained by processing of the 


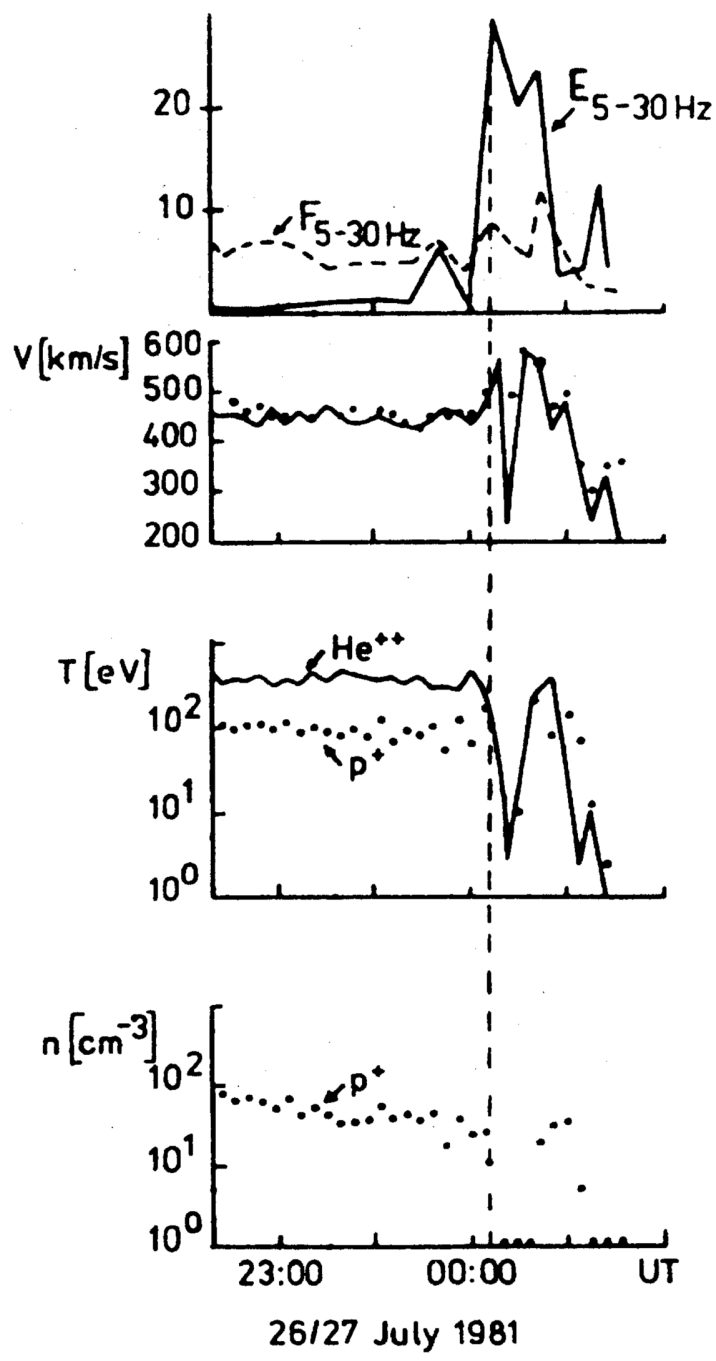

Fig. 5. Plasma parameters and average wave characteristics for the nightside magnetopause and plasma jet close to it. The magnetopause crossing was at 0:08 UT, the plasma cloud with accelerated flow at 0:21 UT.

wave form show similar character of spectra as for the dayside event (Fig. 4). The maxima appear at ioncyclotron frequency and its harmonics. The comparison of the distribution functions as measured by PROGNOZ-8 in the magnetosheath and at the edges of the plasma cloud shows that plasma within this structure is accelerated and distribution function for ions has non-maxwellian tail (Blęcki et al., 1988). The presented observations of the plasma waves associated with the MS-like plasma clouds have been performed during period with perturbed magnetic geomagnetic field (July $27 K_{p}=4$ ).

The summary of the PROGNOZ-8 observation within MS-like plasma clouds below the magnetopause is as follow:

- discrete phenomena with sharp boundaries,

- plasma and magnetic field characteristics similar to those in the dayside FTE's,

- plasma wave activity in e frequency range between ion-cyclotron and lower hybrid,

- $\quad$ possible sources of waves: lower hybrid drift instability, ion-cyclotron instability, ion acoustic beam instability. 


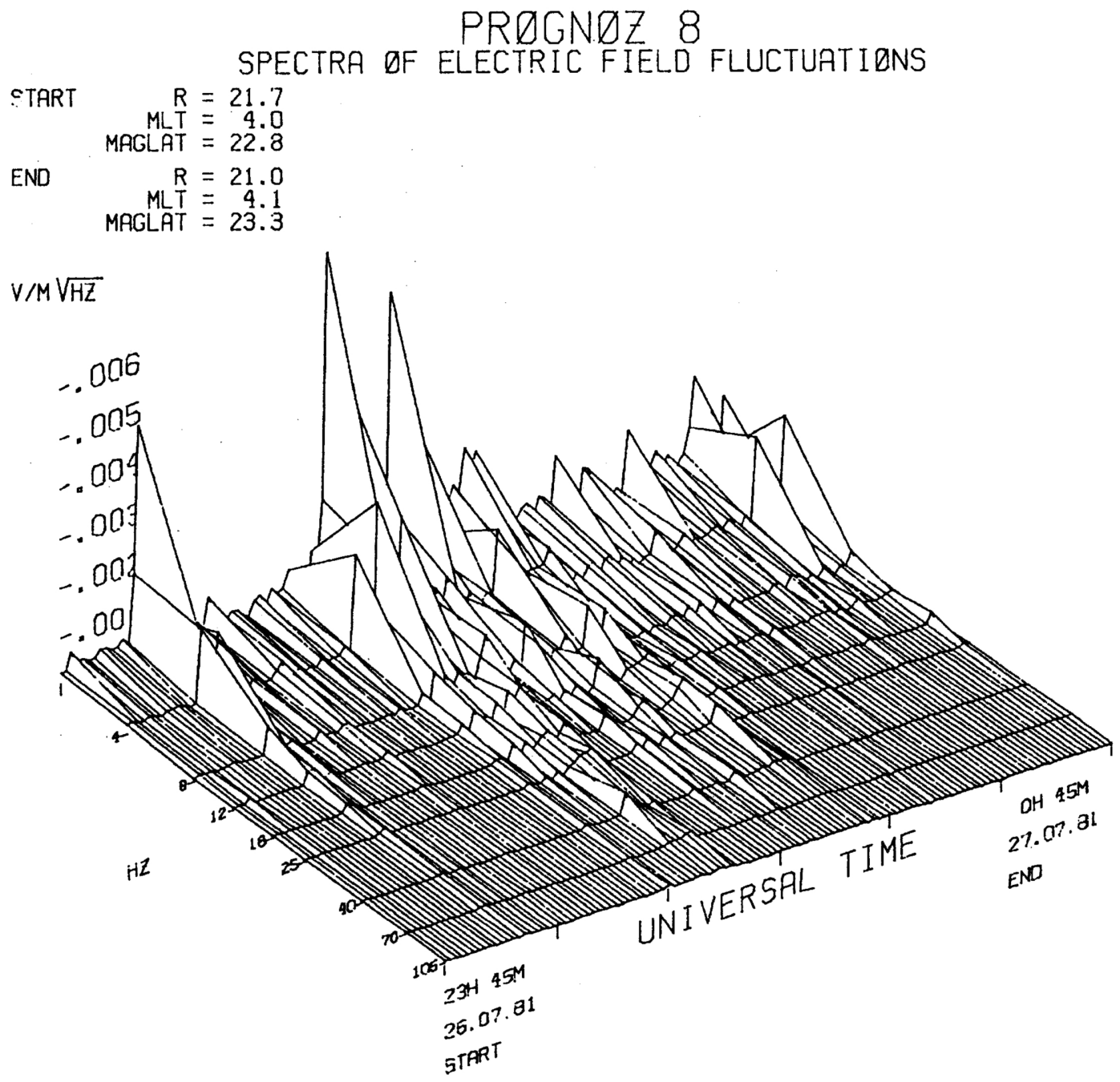

Fig. 6. Detail wave spectra for event shown in Fig. 5.

\section{Magnetotail}

The satellite PROGNOZ-8 was operating in the magnetospheric tail from July up to middle of October 1981. Its orbit allowed us to study a whole cross-section of the magnetospheric tail. An increased level of wave activity in low frequency range was associated with the plasma sheet boundary layer, and hot plasma flow over the PSBL (Klimov et al., 1986).

\subsection{Hot and cold plasma fluxes in the magnetospheric lobe}

The most intense plasma waves in the magnetospheric tail are usually observed in the plasma sheet boundary layer. They have a form of a broad band electrostatic noise (Scarf et al., 1974). Occasionally PROGNOZ-8 crossed plasma structures in the magnetospheric lobe with hot (protons) and cold (oxygen) ion fluxes tailward flowing accompanied by rather cold electrons. 


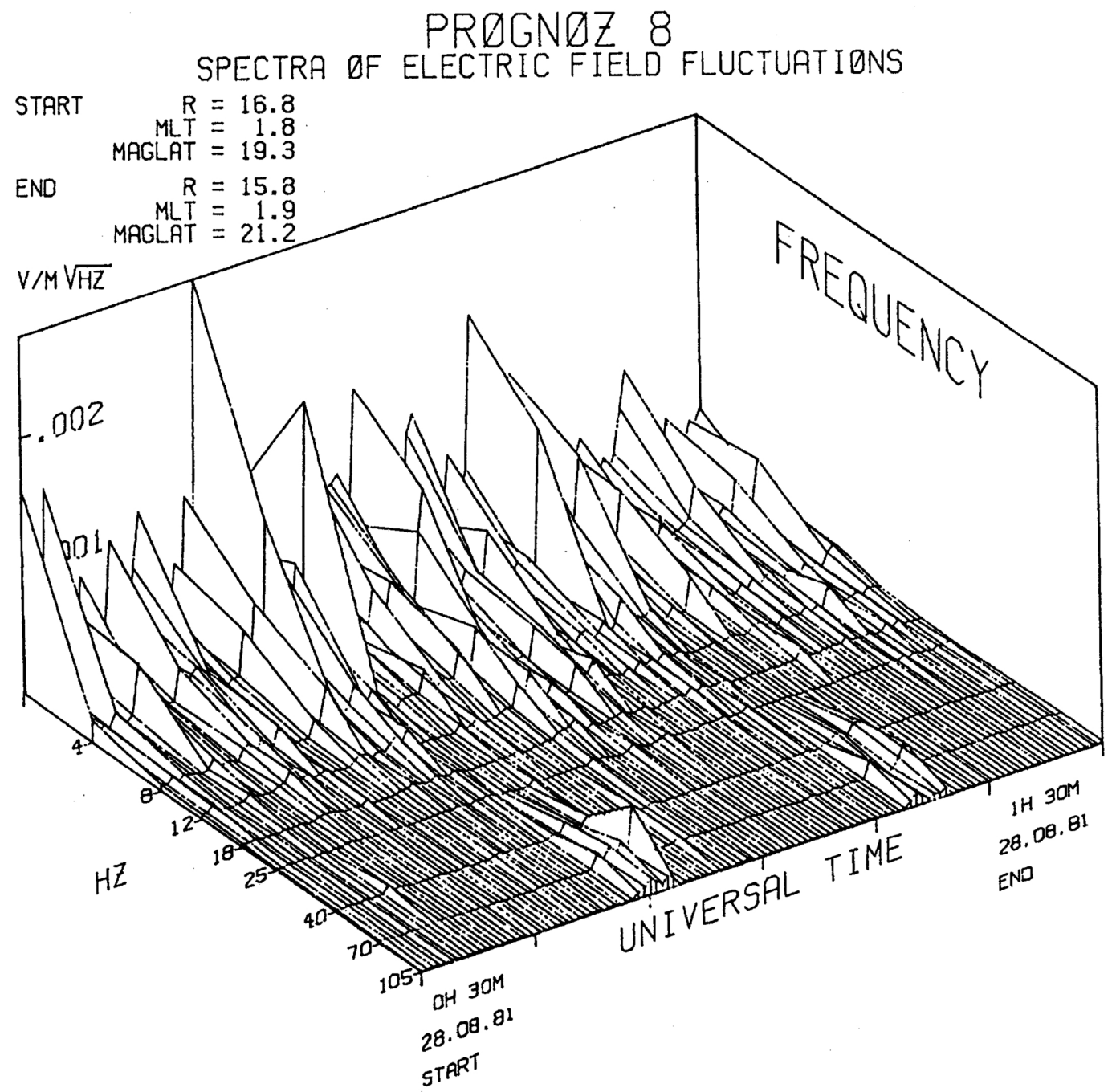

Fig. 7. Wave spectra taken within the hot and cold ion flux (0:50 and 1:15 UT).

These plasma fluxes were different from the regular plasma sheet boundary layer and were associated with strong wave activity in the frequency range $2-70 \mathrm{~Hz}$ (Blęcki et al., 1994). Mentioned above fluxes have been observed on August 28 at 00:50 and 01:15 UT. Strong enhancement of wave activity in the same time can be seen on the spectra presented in Fig. 7. This wave activity can be explain by ion-acoustic beam instability (Blęcki et al., 1994).

\subsection{Plasma sheet boundary layer}

Klimov et al. (1986) studied the integrated over the frequency range $0.1-105 \mathrm{~Hz}$ the plasma wave energy measured in the magnetospheric tail by PROGNOZ-8. The maximum of this wave activity is associated with the PSBL. Figure 8 shows detail wave spectra for detached plasma flux over the PSBL at 3:55 UT and entrance into PSBL at 4:05 UT (Klimov et al., 1986). The broadening of the spectra is associated with this entrance, but the maxima at the lower hybrid frequency $(8-18 \mathrm{~Hz})$ can be seen. 


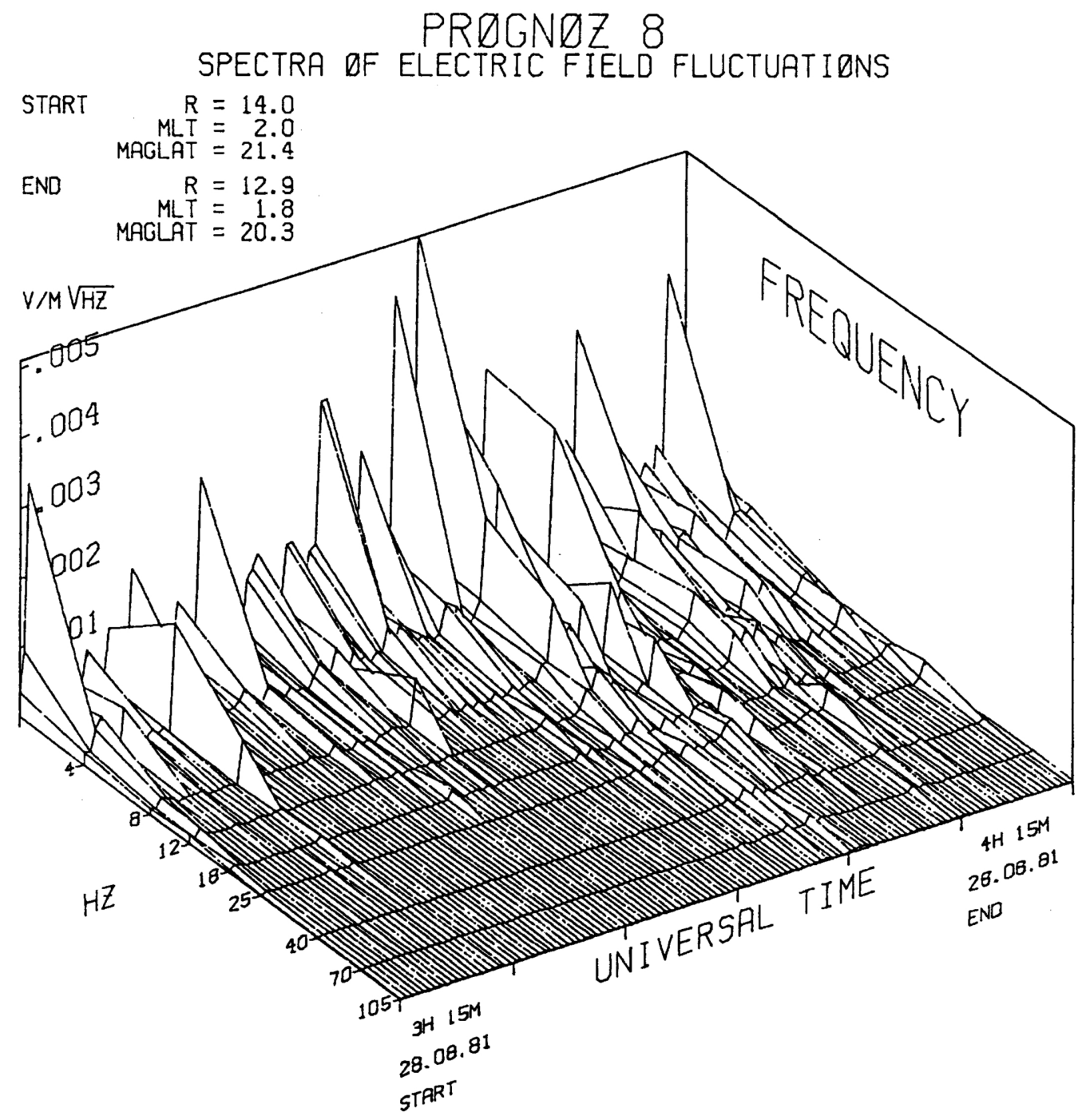

Fig. 8. Wave spectra taken during crossing of the detached from the PSBL plasma flux (3:55 UT) and entrance into PSBL (4:05 UT).

\section{Conclusions}

Presented in this paper wave measurements have been performed by PROGNOZ-8 in different regions of the magnetosphere in rather disturbed geomagnetic situation. The plasma waves in ELF/ULF frequency ranges which play crucial role in the dynamics of the collisionless magnetospheric plasma were observed as typical feature of the active regions. They were observed during magnetospheric substorms, were associated with accelerated plasma jets, with current systems and hot plasma populations. The most intensive waves are associated with ion-cyclotron and lower hybrid modes. Both modes can be a good source of anomalous resistivity which is necessary for reconnection and for plasma heating mechanisms (Treumann et al., 1995). What role play low frequency waves in the reconnection, energy conversion at the bow shock, magnetopause and in a developing of the substorms? This question is still open. We need 
very good simultaneous measurements of wave and particle with high time resolution. We do not have yet the answer for question what is the cause and what is the result in often observed coexistence accelerated plasma and waves, hot population and waves. We need multi-point measurements - in the micro scale and the global scale as well. This chance will be given by CLUSTER together with four spacecrafts of INTERBALL.

This work has been supported by grant KBN 2150P33 and BC-KBN grant WAW/992/007.

\section{REFERENCES}

Anderson, R. R., C. C. Harvey, M. M. Hoppe, B. T. Tsurutani, B. T. Eastman, and J. Etcheto, Plasma waves near the magnetopause, J. Geophys. Res., 87, 2087-2107, 1982.

Belova, E. V., J. Błęcki, M. Denis, L. M. Zeleny, and S. P. Savin, Ion-cyclotron wave generation at the magnetopause, Sov. Plasma Phys., 17, No. 8, 952-961, 1991.

Błęcki, J., K. Kossacki, S. I. Klimov, M. N. Nozdrachev, A. N. Omelchenko, S. P. Savin, and A. J. Sokolov, ELF/ULF plasma waves observed on Prognoz-8 near the magnetopause, Art. Sat. Space Phys., 22, No. 4, 5-16, 1987.

Błęcki, J., K. Kossacki, B. Popielawska, S. I. Klimov, S. A. Romanov, S. P. Savin, and L. M. Zeleny, ELF plasma waves associated with plasma jets near the Earth magnetopause as observed by Prognoz-8, Physica Scripta, 37, 623-631, 1988.

Błęcki, J., M. Denis, K. Kossacki, and L. J. C. Wooliscroft, Plasma waves as a signature of flux transfer events, ESA SP-306, 75$78,1990$.

Błęcki, J., K. Kossacki, R. Wronowski, and D. Zwolakowska, ULF plasma waves observed by Prognoz-8 in the vicinity of the Earth bow shock, ESA WPP-047, 109-112, 1993.

Błęcki, J., B. Nikutowski, P. Koperski, B. Popielawska, K. Kossacki, S. A. Romanov, and S. P. Savin, ELF plasma waves in hot and cold plasma fluxes observed by Prognoz-8 in the magnetospheric tail, Ann. Geophys., 12, 1006-1017, 1994.

Klimov, S. I., S. A. Romanov, M. N. Nozdrachev, S. P. Savin, A. J. Sokolov, L. M. Zeleny, J. Błęcki, K. Kossacki, P. Oberc, B. Popielawska, J. Buchner, and B. Nikutowski, Comparative study of the plasma wave activity in the plasma sheet boundary layer and near Earth plasma sheet, Adv. Space Res., 6, 153-158, 1986.

Paschmann, G., G. Haerendel, N. Scopke, E. Mobius, H. Luhr, and C. W. Carlson, Three-dimensional plasma structures with anomalous flow directions near the Earth's bow shock, J. Geophys. Res., 93, 11279-11294, 1988.

Russell, C. T. and R. C. Elphic, ISEE observations of flux transfer events at the dayside magnetopause, Geophys. Res. Lett., 6, 33-36, 1979.

Sagdeev, R. Z., Cooperative phenomena and shock waves in collisionless plasma, Rev. Plasma Phys., 4, $20-80,1966$.

Scarf, F., L. Franck, K. Ackerson, and Lepping, Plasma wave turbulence at distant crossings of the plasma sheet boundaries and neutral sheet, Geophys. Res. Lett., 1, 189-192, 1974.

Schwartz, S. J., R. L. Kessel, C. C. Brown, L. J. C. Woolliscroft, M. W. Dunlop, C. J. Farrugia, and D. S. Hall, Active current sheets near the Earth's bow shock, J. Geophys. Res., 93, 11295-11310, 1988.

Thomsen, M. F., J. T. Gosling, S. A. Fuselier, S. J. Bame, and C. T. Russell, Hot diamagnetic cavities upstream from the Earth's bow shock, J. Geophys. Res., 91, 2961-2973, 1986.

Treumann, R. A., J. LaBell, and T. M. Bauer, Diffusion processes: an observational perspective, in Physics of the Magnetopause, edited by P. Song, B. U. O. Sonnerup, and M. F. Thomsen, pp. 331-342, American Geophysical Union, Washington, D.C., 1995.

Vaisberg, O. L., A. A. Galeev, G. N. Zastenker, S. I. Klimov, M. N. Nozdrachev, R. Z. Sagdeev, A. J. Sokolov, and V. D. Shapiro, Electron acceleration in the front of strong collisionless shock wave, Sov. Phys. JETP., 58, 4, 1983.

Woolliscroft, L. J. C., S. J. Schwartz, C. C. Brown, C. P. Chaloner, P. J. Christiansen, A. Coates, A. G. Darbyshire, M. P. Gough, D. S. Hall, A. D. Johnstone, W. A. C. Mier-Jedrzejowicz, A. J. Norris, R. P. Rijnbeek, and D. J. Southwood, AMPTE-UKS observations of current sheets in the solar wind, Adv. Space Res., 6, No. 1, 89-92, 1986. 